\title{
Acute effects of exercise intensity on subsequent appetite, energy intake, resting energy expenditure and 24 hour blood pressure
}

\author{
R. Bracegirdle, H. Finck and A. Collins \\ Division of Nutritional Sciences, Faculty of Health and Medical Sciences, University of Surrey, Guildford, GU2 7XH, UK
}

The UK has a high prevalence of obesity and hypertension, both of which are well established cardiovascular disease (CVD) risk factors. The extent to which exercise regulates components of energy balance, including post-exercise appetite, energy intake, resting energy expenditure (REE) and hence resulting weight is not fully understood. However, exercise can confer health benefits such as reductions in $\mathrm{BP}$, independent of weight change ${ }^{(1)}$. Although inconclusive at present, it's been suggested the intensity of exercise encountered may influence subsequent energy balance ${ }^{(2)}$ and $\mathrm{BP}^{(3)}$. This study aimed to examine this theory.

Ethical approval was received from the Faculty of Health and Medical Sciences, University of Surrey. Eight female subjects (18-24 yrs, $58.2 \pm 8.4 \mathrm{~kg}, 1.60 \pm 0.08 \mathrm{~m}$ ) underwent two exercise conditions, high intensity (HI) and moderate intensity (MI), with a minimum 5 day wash-out period in-between each condition. $\mathrm{HI}$ was undertaken as interval exercise with subjects completing 8 bouts at $98.9 \pm 7.75 \% \mathrm{VO}_{2}$ max. MI consisted of 26 minutes continuous exercise at $61.6 \pm 12.0 \% \mathrm{VO}_{2}$ max. Visual analogue scales (VAS) were used to subjectively record appetite pre-exercise and at 30 minute intervals for a further 240 minutes post-exercise. Ad-libitum energy intake was assessed at a test meal and ' 24 hour' energy intake was recorded via food diaries. BP was recorded every 30 minutes during daytime hours, and every hour during the night, for an average of 22 hours post-exercise. REE was measured by indirect calorimetry the following day. Paired samples t-test was performed on all variables to detect if there were any significant differences in the variables tested, between the two exercise conditions.

No significant difference was found between HI and MI exercise for variables which modify energy balance; post-exercise appetite $(P \geq 0.477)$, energy intake $(P \geq 0.556)$ and REE $(P \geq 0.958)$ or for $\mathrm{BP}$, measured for an average of 22 hours post exercise $(P \geq 0.155)$.

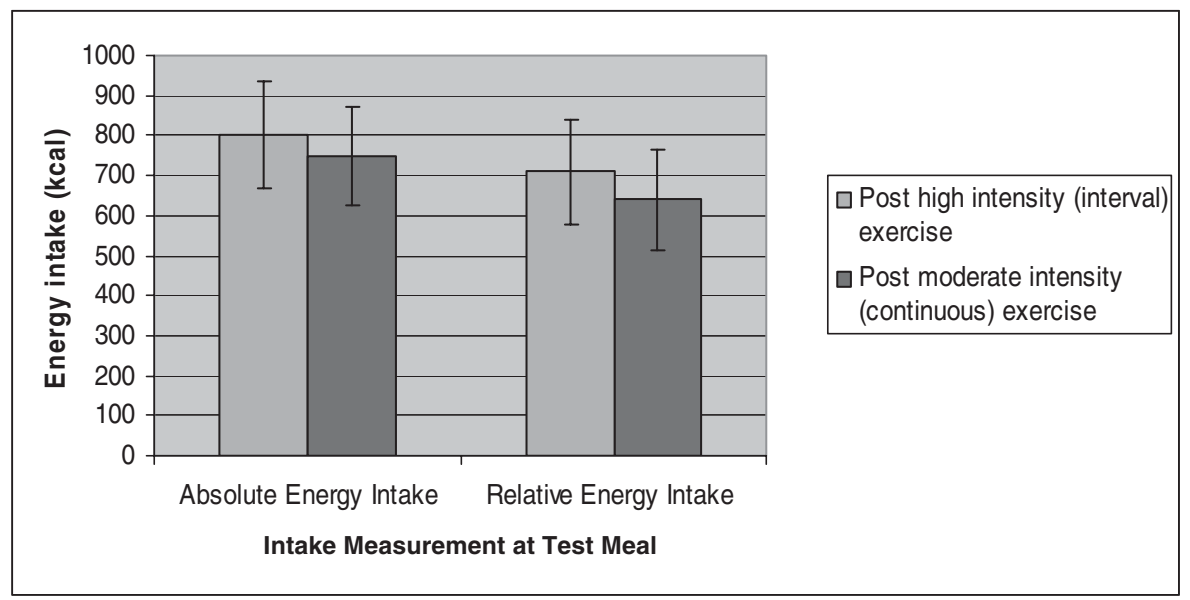

Fig. 1. Mean 'absolute' and 'relative' energy intake (kcal) at test meal provided 2 hours post HI (interval) and MI (continuous) exercise

This study shows there are no significant acute differences between HI and MI exercise on post-exercise appetite, energy intake, REE and 24 hour BP in lean, normotensive females. These acute findings do not support the recommendation of one exercise intensity over another for regulation of energy balance/CVD risk. The conclusions of this study are that any recommendations concerning the appropriate type of exercise intensity to perform should be based on factors such as individual's preference, rather than reasons concerning differences in provision of health benefits. However, for clarification of these conclusions, a study investigating the chronic effects of exercise intensity on these variables is warranted.

1. Hopkins M, King NA \& Blundell JE (2010) Curr Opin Clin Nutr Metab care 13, 635-640.

2. Ueda SM, Yoshikawa T, Katsura Y et al. (2009) J Endocrin 203, 357-364.

3. Cornelissen VA, Verheyden B \& Aubert AE (2010) J Hum Hypertens 24, 75-82. 